\title{
VARIÉTÉ, EMPLOI ET CHÔMAGE AUX PAYS-BAS
}

Koen Frenken, Thijs Verburg et Frank G. van Oort

OFCE | « Revue de l'OFCE »

2008/1 n $104 \mid$ pages 203 à 218

ISSN 1265-9576

ISBN 9782724631104

Article disponible en ligne à l'adresse :

https://www.cairn.info/revue-de-l-ofce-2008-1-page-203.htm

Distribution électronique Cairn.info pour OFCE.

(C) OFCE. Tous droits réservés pour tous pays.

La reproduction ou représentation de cet article, notamment par photocopie, n'est autorisée que dans les limites des conditions générales d'utilisation du site ou, le cas échéant, des conditions générales de la licence souscrite par votre établissement. Toute autre reproduction ou représentation, en tout ou partie, sous quelque forme et de quelque manière que ce soit, est interdite sauf accord préalable et écrit de l'éditeur, en dehors des cas prévus par la législation en vigueur en France. Il est précisé que son stockage dans une base de données est également interdit. 


\section{VARIÉTÉ, EMPLOI ET CHÔMAGE AUX PAYS-BAS *}

\section{Koen Frenken et Thijs Verburg}

Centre de Recherche Urbaine et Régionale Utrecht (URU), Université d'Utrecht

\section{Frank G. van Oort}

Institut néerlandais de Recherche Spatiale (RPB), La Haye et URU

Dans la théorie économique, la variété de production peut se présenter de manière distincte suivant qu'elle constitue une source de retombées de connaissances régionales, appelées externalités de Jacobs, ou un portefeuille qui protège la région des chocs externes. Nous affirmons que la variété intrasectorielle (au sein des secteurs) permet de mieux mesurer les externalités de Jacobs alors que la notion de portefeuille est mieux saisie par la variété intersectorielle (entre les secteurs). Nous présentons une méthodologie fondée sur des mesures d'entropie afin d'estimer ces deux types de variété. À partir des données régionales des Pays-Bas sur la période 1996-2002, nous constatons que les externalités de Jacobs augmentent la croissance de l'emploi, alors que la variété intersectorielle atténue celle du chômage. S'ensuivent des implications pour la politique régionale.

Mots clés : Variété de production. Marché du travail.

* Cette recherche a été financée par le ministère des Affaires économiques des Pays-Bas dans le cadre du programme de recherche sur « l'Espace et la productivité ». Nous tenons à remercier Herm van der Beek, Ron Boschma, Henri de Groot et Jacco Hakfoort pour l'aide qu'ils nous ont apportée à travers leurs commentaires sur des versions antérieures. Nous remercions également Bart Los pour les données sur les similarités technologiques entre secteurs.

k.frenken@geo.uu.nl

oort@rpb.nl

thijsverburg@hotmail.com 
I a relation entre la variété et le développement économique est un domaine de recherche négligé en économie. Depuis longtemps, la théorie économique s'est attachée à expliquer l'économie en combinant la croissance des inputs avec celle des rendements (Solow, 1957). La nature qualitative du développement économique, par exemple en termes de variété des secteurs ou des technologies, n'a que rarement été abordée.

Dans cet article, nous analysons l'effet de la variété de secteur sur la croissance régionale de l'emploi et du chômage aux Pays-Bas. Le principal argument est qu'à travers différents mécanismes, la variété, à la fois, stimule la croissance de l'emploi et protège des chocs de demande. En introduisant les concepts de variété intrasectorielle et de variété intersectorielle, nous montrons que la première affecte positivement la croissance de l'emploi, tandis que la seconde - tel un portefeuille - atténue l'effet des chocs. Nous poursuivons alors notre réflexion par une discussion des politiques actuelles.

\section{Cadre théorique}

\section{I.I. Les externalités de Jacobs}

Au moins deux types de relations peuvent être distingués entre la variété et le développement économique. La première approche se focalise sur la variété, les externalités et la croissance, des thèmes devenus centraux dans ce qui est appelé « la nouvelle théorie de la croissance ». II est admis que, dans un cadre statique, la variété de secteur est plus élevée en milieu urbain qu'en milieu rural. La présence de marchés plus vastes et plus intenses, tout comme les opportunités d'économies d'échelle internes, expliquent que les firmes et les consommateurs préfèrent se localiser dans une ville centrale laissant ainsi l'agriculture, le tourisme et les services fondamentaux en périphérie (Krugman, 1991). Suivant l'approche de la nouvelle géographie économique, la variété est uniquement le résultat des décisions des agents économiques de se regrouper ; elle n'est pas considérée comme une source de croissance économique. Plus précisément, la variété ne pourrait être une source d'externalités géographiquement localisés renforçant la croissance économique régionale (Glaeser et al., 1992 ; Feldman et Audretsch, 1999 ; Acs, 2002), telles que Jane Jacobs (1969) appelle les externalités de Jacobs. Autrement dit, les retombées écono- 
miques pourraient survenir essentiellement quand une région est spécialisée dans quelques secteurs (économies de localisation) ou si elle est principalement liée au niveau de densité de la population (économies d'urbanisation). Les externalités de Jacobs, les économies de localisation et les économies d'urbanisation sont des types d'économies d'agglomération résultant de la concentration géographique de l'activité économique. En principe, elles peuvent se produire simultanément dans la mesure où une entreprise peut apprendre de plusieurs firmes appartenant à la même industrie (économies de localisation), de plusieurs firmes évoluant dans des industries différentes (externalités de Jacobs), ou de la concentration en soi (économie d'urbanisation).

La question de savoir si la croissance régionale profite davantage des économies de localisation ou des externalités de Jacobs pose également le problème voisin qui est celui de la composition des secteurs. Étant donné que le niveau des spillovers diffère, à la fois au sein de chaque secteur et entre chaque paire de secteurs, la question est de déterminer quelle composition précise des secteurs, dans une économie régionale, engendre le plus d'externalités. Idéalement, une économie régionale est spécialisée dans les secteurs qui sont liés à un secteur dont la R\&D investie se diffuse alors à ces derniers. La distinction entre les différentes sources d'effets externes induit d'importantes implications dans la théorie, car les diverses catégories d'externalités devraient conduire à des types qualitativement différents d'avantages. Les économies de localisation devraient stimuler l'innovation incrémentale et le processus d'innovation, étant donné que les connaissances qui se diffusent émanent de firmes similaires produisant des produits similaires. L'impact des économies de la localisation devrait alors s'exprimer principalement par l'accroissement de la productivité. En revanche, les externalités de Jacobs devraient favoriser des innovations plus radicales et des innovations de produit dans la mesure où les connaissances et les technologies des différents secteurs sont recombinées afin de concevoir de nouveaux produits ou de nouvelles technologies (comparable au concept de Schumpeter de Neue Kombinationen). De plus, puisque des innovations radicales et des innovations de produit conduisent à la création de nouveaux marchés et à de l'emploi, plutôt qu'à des hausses de productivité, leur impact peut être très différent de celui des innovations incrémentales et de processus induites par les économies de localisation (cf. Saviotti et Pyka, 2004 a,b).

\section{I.2. Théorie du portefeuille}

Une seconde manière de relier la variété au développement économique régional, et plus précisément au chômage, est de considérer la variété comme une stratégie de portefeuille visant à protéger une région des chocs externes de demande (Attaran, 1985 ; Dissart, 2003). Dans 
cette optique, on peut également parler de diversification régionale analogue à la diversification d'entreprise. Comme pour les entreprises, la diversification peut être considérée comme une stratégie de répartition des risques pour une région. Pour une économie régionale, une grande diversification sectorielle fait qu'un choc négatif de ses secteurs n'aura que des effets faibles sur la croissance et l'emploi. Au contraire, une région peu diversifiée, se spécialisant dans un secteur ou un groupe de secteurs corrélés à la demande, court le risque de voir survenir un sérieux ralentissement de la croissance et un taux élevé de chômage à la suite d'un choc de demande. La variété protégera particulièrement le marché du travail et empêchera donc la forte augmentation du chômage. Même si la mobilité inter-régionale du travail est élevée, empêchant la hausse du chômage, des chocs asymétriques diminuent la croissance économique et détériorent les économies d’agglomération et l'assiette fiscale (Krugman, 1993). Dans ce cadre, la variété industrielle au niveau régional permettrait de réduire le chômage régional et de promouvoir la croissance économique régionale, tandis que la spécialisation augmenterait le risque de chômage et d'un ralentissement de la croissance.

À la différence des secteurs sans rapport les uns avec les autres, on peut s'attendre à ce que des secteurs liés soient davantage corrélés aux chocs de demande. Par conséquent, au regard de la stratégie de portefeuille, le risque de propagation aux secteurs non liés est plus faible que le risque de propagation aux secteurs liés. Les effets de la variété de secteur suivant que cette dernière est intra ou intersectorielle diffèrent donc. La variété intersectorielle protège mieux une région des chocs asymétriques de demande. En revanche, la variété intrasectorielle devrait être bénéfique aux économies d'urbanisation et aux externalités de connaissances, renforçant ainsi la croissance et l'emploi. Ceci nous conduit aux hypothèses suivantes :

Hypothèse 1 : La variété intrasectorielle affecte positivement la croissance de l'emploi régional

Hypothèse 2 : La variété intersectorielle protège des chocs de demande et de la croissance du chômage régional

\section{Méthodologie}

\section{I. Variables dépendantes}

La croissance de l'emploi (1996-2002) est calculée en pourcentage de croissance des employés équivalents temps-plein (ETP) (1996-2002) en utilisant des données émanant du Bureau Central des Statistiques 
néerlandais (CBS). Ces données d'emploi sont également utilisées pour construire les variables en ETP où les niveaux d'emploi sont utilisées comme dénominateur (salaire en ETP, investissement en ETP, R\&D en ETP, voir ci-dessous). On peut noter que ces données incluent toutes les activités économiques à l'exception de l'agriculture.

La croissance du chômage (1996-2002) est calculée en pourcentage de croissance (1996-2002) à partir des données du CBS. Nous avons également utilisé la croissance de l'inactivité en tant qu'indicateur alternatif de la croissance du chômage. L'inactivité fait référence à la fois au nombre de chômeurs et au nombre de travailleurs invalides physiquement (souvent perçus comme une forme cachée de chômage, en particulier aux Pays-Bas).

\subsection{Variables indépendantes}

La variété intersectorielle (1996). Un problème de mesure important est de savoir comment mesurer et faire la différence entre la diversification intra et intersectorielle. Comme cela a été précisé précédemment, le concept de variété intrasectorielle repose sur l'idée que certains secteurs sont davantage liés que d'autres et généreront relativement plus d'externalités de connaissances inter-industrie. Une mesure de la diversification intrasectorielle et intersectorielle, à la fois au niveau de la firme (Jacquemin et Berry, 1979) et au niveau régional (Attaran, 1985), réside dans le calcul de l'entropie (Theil, 1967). Son principal avantage, et la raison de son utilisation dans le contexte de la diversification, sont que l'entropie peut être décomposée à chaque niveau de classification sectorielle. Cela permet de mesurer l'effet de la variété à différents niveaux d'agrégation. La nature décomposable de l'entropie implique que la variété, à plusieurs niveaux de classification sectorielle, peut être l'objet d'une analyse de régression sans induire nécessairement de colinéarité (Jacquemin et Berry, 1979 ; Attaran, 1985). Nous définissons la variété intersectorielle par l'indice d'entropie de la décomposition sectorielle à 2 chiffres. Quant à la variété intrasectorielle, nous la définissons par l'augmentation marginale de l'entropie lorsque le niveau d'agrégation de la classification sectorielle passe de 2 à 5 chiffres. Ainsi, la variété intrasectorielle mesure la variété au sein de chaque classe industrielle à deux chiffres. Par exemple, une région produisant uniquement des services, mais toutes les variétés de services, présentera une faible variété intersectorielle et une variété intrasectorielle élevée. II est important de souligner que les valeurs de l'entropie pour les deux types de variétés ne sont pas corrélées (la corrélation est égale à - 0,042 et n'est pas significative). Cela montre déjà que la distinction entre les variétés intrasectorielle et intersectorielle est statistiquement instructive. Les données régionales sur l'emploi sectoriel, au niveau de la classification à cinq chiffres, sont fondées sur les données 
d'emploi qui proviennent de la base de données LISA sur l'emploi aux Pays-Bas (des données retravaillées de Van Oort, 2004).

Indice de Los de Fabrication (1996). Les économies de localisation sont associées à la concentration d'un secteur particulier dans une région. Nous avons construit une variable, l'indice de Los (Los, 2000). Cet indice exprime la parenté technologique entre les secteurs industriels en calculant la similarité entre l'input de deux secteurs associée à un tableau d'entrées-sorties (voir Frenken et al., 2004 pour plus de détails).

Logarithme de densité de population (1996). La densité de population est utilisée comme un indicateur approximatif des économies d'urbanisation résultant d'une importante concentration de l'activité économique (c'est-à-dire quelle que soit sa composition). Le principal élément des économies d'urbanisation réside dans les bénéfices provenant de la taille de marché estimée par la densité de population. Nous avons choisi de prendre le logarithme de cette variable exprimant la baisse du profit marginal de chaque habitant supplémentaire dans une région.

Salaire (1996). Provenant du Bureau central de statistiques (CBS) pour l'année 1996, les niveaux de salaire moyen sont utilisés comme une variable de contrôle des différentiels de croissance de l'emploi résultant des différentiels de salaire.

Investissement en ETP (moyenne 1996-2002). Les données concernent les investissements en biens d'équipements fixes, à l'exception des habitations. L'indicateur est calculé en équivalent temps plein (ETP) et les données sont extraites des statistiques néerlandaises (CBS).

Taux de croissance du ratio capital-travail (1996-2001). La croissance du ratio capital-travail (croissance C-T) est calculée en pourcentage de croissance (1996-2001) et a pour origine l'étude de la productivité régionale de Broersma et Oosterhaven (2004). Cette variable est principalement destinée à expliquer la croissance de la productivité. Suivant l'approche de la fonction de production (Solow, 1957), une augmentation de capital par rapport au travail contribuera à la productivité du travail. La variable croissance C-T peut également être interprétée comme résultant d'une innovation de processus, prise au sens large, ce qui devrait contribuer à la productivité du travail (Kim, 1997).

$R \& D$ en ETP (1999). Les données sur la Recherche et Développement (R\&D) font référence au niveau d'investissements en R\&D en 1999 (les données de l'année 1996 n'étant pas disponibles). Les données proviennent de Senter (Van Oort, 2004).

Croissance des zones d'activité (1996-2002) et croissance des habitations (1996-2002). Les quartiers résidentiels récemment construits et 
les locaux commerciaux attirent l'activité économique qui, auparavant, n'était pas présente à cet endroit. Pour contrôler ces causes potentielles d'une croissance extrêmement élevée de l'emploi différentiel, nous introduisons la croissance des sites d'activités mesurée en hectares (moyenne 1996-2002) et la croissance du nombre d'habitations pour la même période. Ces informations proviennent des statistiques néerlandaises (CBS).

Randstad (RS) et Périphérie nationale (PER). Des recherches antérieures (Van Oort, 2004), nous ont appris que l'hétérogénéité spatiale peut apparaître au niveau des deux groupes de régions des Pays-Bas : la région centrale (que l'on nomme aux Pays-Bas la Randstad et qui constitue une conurbation des provinces de l'ouest réunissant des villes comme Amsterdam, Haarlem, Leiden, Delft, Rotterdam et Utrecht) ou la périphérie (les provinces du nord, de l'est et du sud). Nous utilisons la distinction entre le centre et la périphérie en effectuant les régressions séparément pour les deux zones.

Les statistiques descriptives sont présentées dans le tableau 1. Les valeurs indiquées sont les valeurs répondant aux définitions présentées dans la section précédente. Par la suite, nous utilisons les résultats normalisés (cote $z$ avec une moyenne de 0 et un écart-type de 1) de toutes les variables afin d'évaluer l'effet relatif des variables indépendantes. Certaines variables ont été corrigées en tant que témoin (voir Frenken et al., 2004). Comme principales variables de contrôle, nous avons choisi d'introduire investissement et $R \& D$. En outre, quand il s'agit de la croissance du chômage, nous introduisons la variable croissance du ratio capital-travail comme variable de contrôle. Une augmentation du ratio capital-travail peut résulter d'un changement technologique réduisant le recours au travail, susceptible d'accroître le chômage. Enfin, nous avons également introduit la variable salaire dans notre modèle de base afin d'expliquer la croissance du chômage, dans la mesure où les régions présentant des niveaux de salaire relativement plus élevés devraient connaître un niveau plus important de chômage, toutes choses égales par ailleurs. Toutes les autres variables sont introduites une à une dans le modèle de référence afin de déterminer si la spécification du modèle s'améliore. Si tel est le cas, ces variables sont présentées dans les résultats. 
I Koen Frenken, Frank G. van Oort et Thijs Verburg

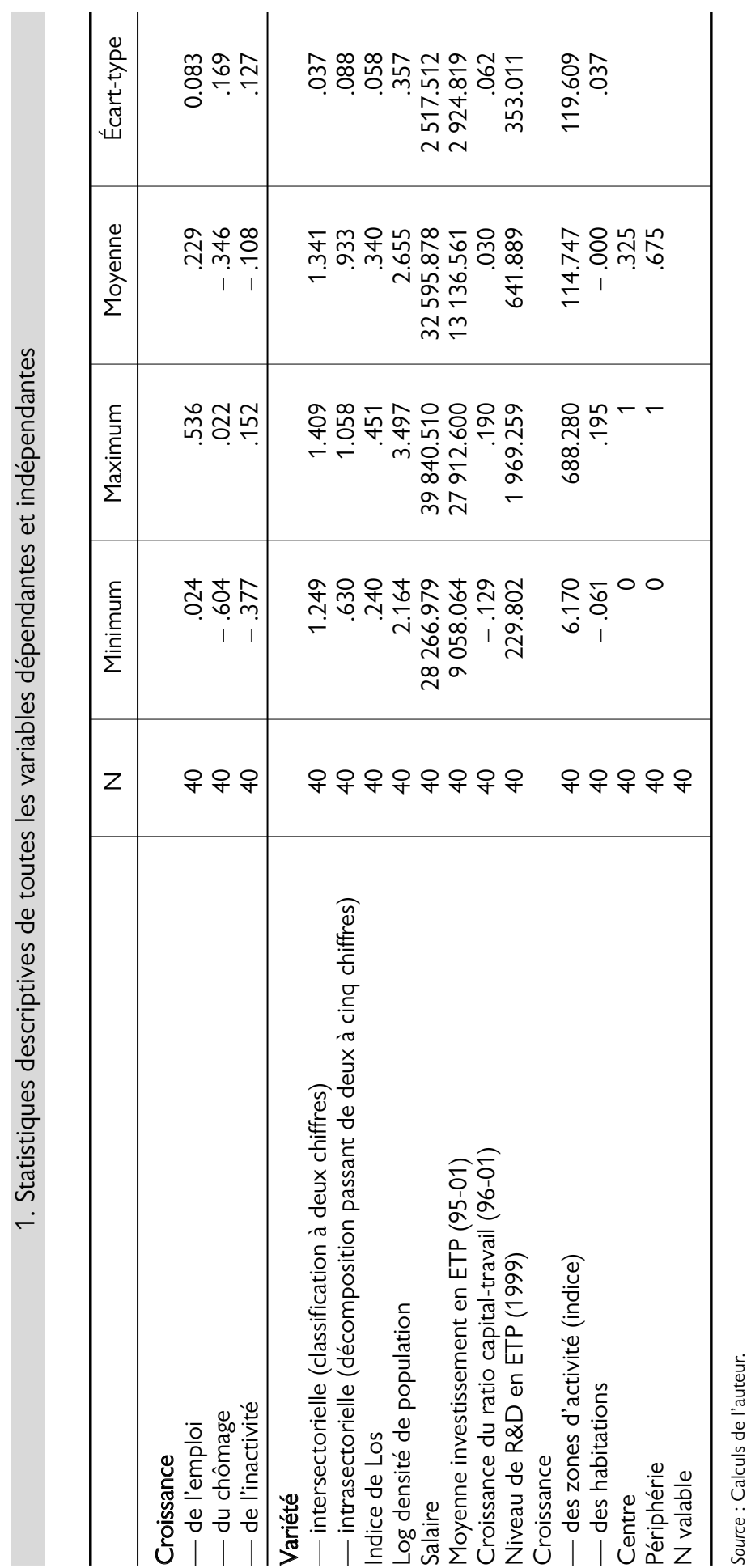




\section{Résultats}

\section{I. Résultats concernant la croissance de l'emploi}

Le tableau 2 présente les résultats pour ce qui a trait à la variable croissance de l'emploi, en tant que variable dépendante. Le modèle 1 spécifie le modèle $M C O$ de référence. D'après les résultats, on peut conclure que l'hypothèse 1 est confirmée : la variété intrasectorielle en tant qu'indicateur des externalités de Jacobs est positivement et significativement liée à la croissance de l'emploi ${ }^{1}$. Dans la mesure où nous utilisons les cotes-z, les résultats mettent en évidence que la variété intrasectorielle contribue également davantage à la croissance de l'emploi. En outre, l'investissement en tant que variable de contrôle constitue un signal attendu. II est intéressant de noter que la densité de population n'a pas d'effet significatif sur la croissance de l'emploi, suggérant que ce n'est pas l'urbanisation qui contribue à la création d'emplois mais la variété intrasectorielle (qui est corrélée à la densité de population, voir tableau 2). En d'autres termes, les «villes» ne créent pas d'emplois « automatiquement ». La variété intrasectorielle est plutôt responsable de la création d'emplois qui sont souvent, mais pas nécessairement, de plus hauts niveaux dans les villes.

Les modèles $1 \mathrm{a}$ et $1 \mathrm{~b}$ testent la robustesse du modèle 1 , en substituant la variable dépendante " croissance de l'emploi » au cours de la période 1996-2002, par la même variable mais à des périodes différentes, soient (1997-2001) et (1996-2001). Les résultats montrent que le modèle 1 est robuste au sens où les mêmes variables sont significatives (et de même signe) dans les modèles $1 \mathrm{a}$ et $1 \mathrm{~b}$ que dans le modèle 1.

En utilisant le modèle 1, nous avons ajouté, une à une, toutes les autres variables. Aucune d'entre elles ne s'est révélée être significativement liée à la croissance de l'emploi, excepté pour le niveau de salaire moyen (modèle 2 ) et la croissance des habitations (modèle 3 ). Dans les deux cas, l'investissement n'était pas très significatif mais la significativité et le signe de la variété intrasectorielle prouvent qu'elle est robuste. Le modèle 2 suggère que l'emploi a été créé dans des zones à salaires élevés. Ceci est en contradiction avec ce que l'on trouve traditionnellement, à savoir que les faibles niveaux de salaires attirent l'investissement et, ce faisant, créent des emplois. On peut noter que la variable salaire rend la densité de population significative

1. Nous avons également estimé le modèle de référence sans la variable variété intersectorielle et sans la variable variété intrasectorielle, tout en introduisant une entropie selon la classification sectorielle à cinq chiffres plutôt qu'un vaste indicateur de la « variété totale ». Ceci n'a pas modifié les résultats. 
et négative (en raison de la corrélation relativement élevée entre les salaires et la densité de population). Le modèle 3 est pertinent car il montre les effets de la croissance des habitations sur la croissance de l'emploi. Ceci renvoie à la question de savoir si les investissements dans les logements attirent les emplois (« le travail suivant les conditions de vie ») ou si c'est l'inverse (« les conditions de vie suivant le travail »). La croissance de l'emploi devrait être tributaire des habitations, de nouveaux habitants attirant de nouveaux emplois dans la plupart des secteurs de service ${ }^{2}$.

Nous avons également effectué des régressions $M C O$ séparément pour la région centrale et la périphérie (modèle 4). Dans les deux cas, le test de Chow-Wald révèle que les différences entre les zones ne sont pas significatives. Cependant, les effets des variables individuelles peuvent être significativement différents dans les régions centrales ou périphériques. Seuls les niveaux d'investissements montrent une relation différente avec la croissance de l'emploi dans les différentes situations, leurs effets étant significativement positifs dans la Randstad, tout en n'étant pas significatifs dans le reste du pays.

\subsection{Résultats pour la croissance du chômage}

Le tableau 3 présente les résultats pour la variable croissance du chômage et la variable croissance de l'inactivité en tant que variables indépendantes. Le modèle 1 spécifie le modèle $M C O$ de référence. À partir des résultats, on peut conclure que l'hypothèse 2 concernant la croissance du chômage — la variété intersectorielle est négativement liée à la croissance du chômage — est validée. Ceci signifie que les régions présentant une variété intersectorielle plus élevée ont des taux plus faibles de croissance du chômage. En outre, nous constatons une relation significativement négative entre les économies d'urbanisation et la croissance du chômage. Ceci peut être expliqué par le fait que les régions caractérisées par des hautes densités de population sont également des régions où les chômeurs disposent de davantage d'opportunités de travail au sein d'une gamme d'emplois substituables. Les économies d'urbanisation offrent, par conséquent, une garantie contre une importante croissance du chômage. Nous trouvons également que les régions caractérisées par des dépenses de R\&D en ETP et une croissance C-T élevées présentent une croissance du chômage plus forte, ce qui suggère qu'une partie de l'activité d'innovation a vu ses besoins en facteur travail réduits. Enfin, nous constatons l'effet attendu des salaires sur le chômage.

2. Ceci laisse suggérer la présence d’endogénéité. 


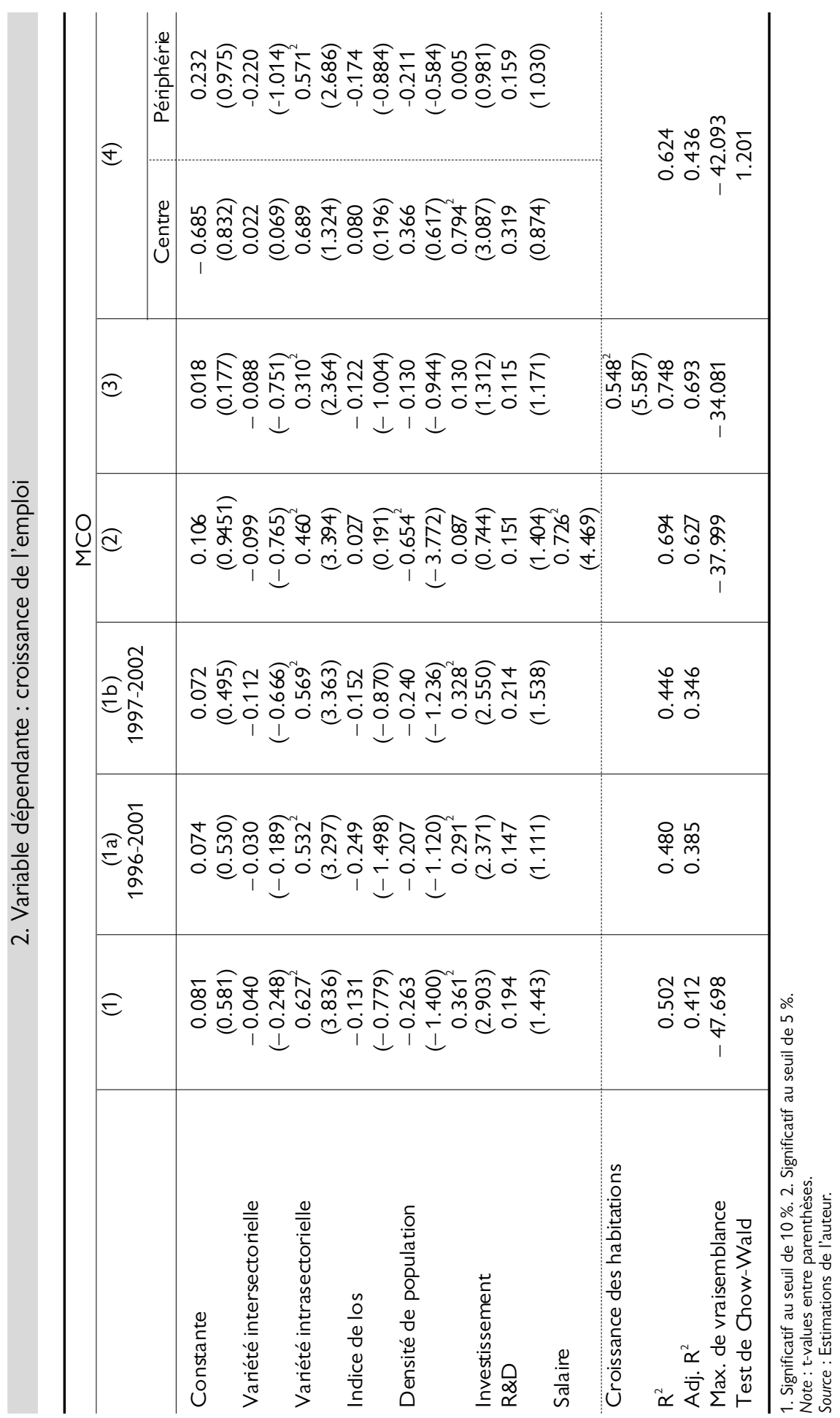


I Koen Frenken, Frank G. van Oort et Thijs Verburg

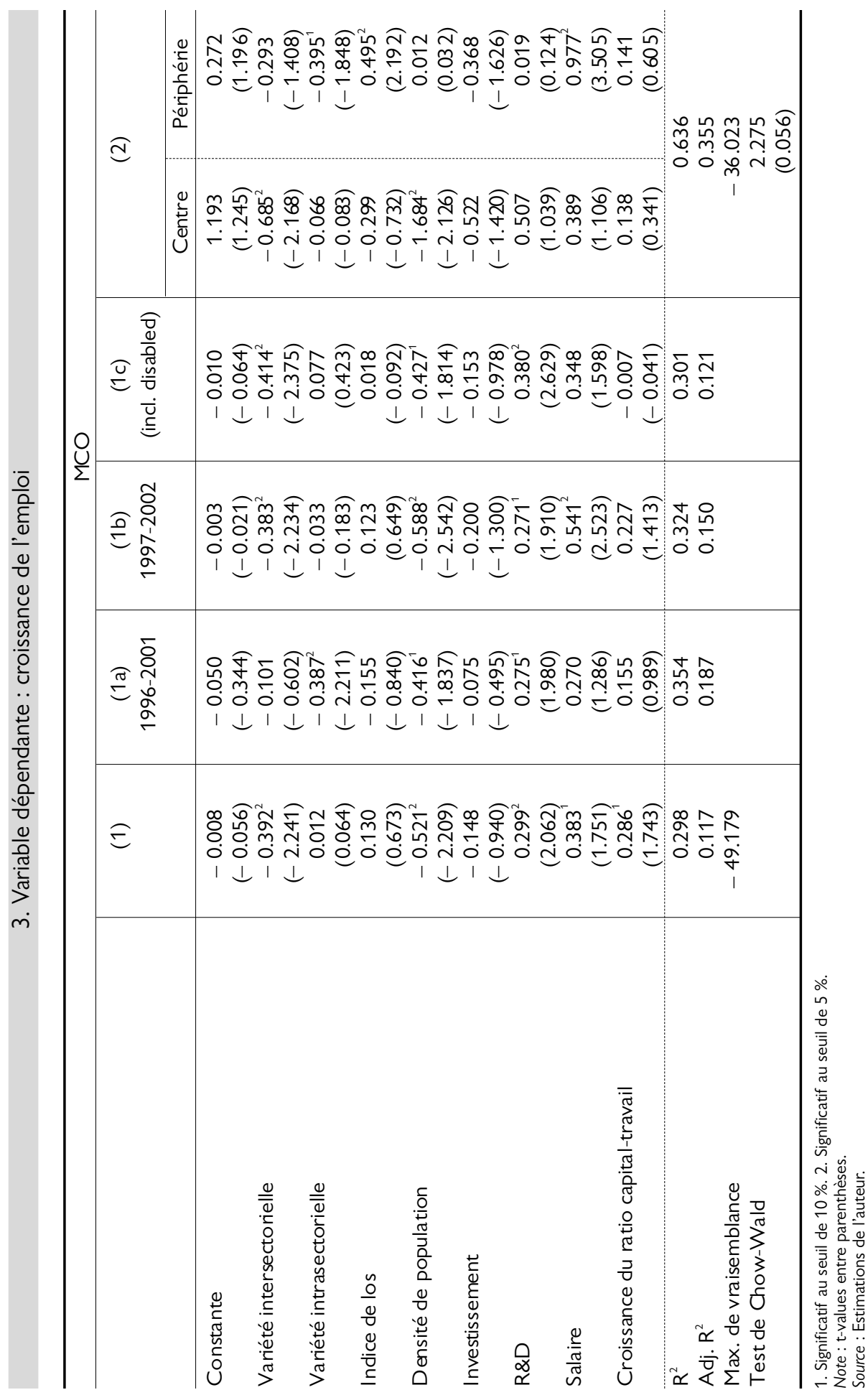


Les modèles $1 \mathrm{a}$ et $1 \mathrm{~b}$ testent la robustesse du modèle 1 en substituant la croissance du chômage au cours de la période 1996-2002 par la même variable mais à des périodes différentes (1997-2002) et (19962001). Le modèle 1c apporte une robustesse supplémentaire en ajoutant la variable croissance de l'inactivité (en incluant les invalides physiques dans la définition de l'inactivité, en plus des chômeurs) comme alternative pour mesurer le chômage à la même période. Les résultats sur la robustesse montrent que le modèle de référence n'est pas entièrement fiable dans le cas de changements dans la période d'observation, étant donné que la variété intersectorielle, le salaire et la croissance $\mathrm{C}-\mathrm{T}$ sont significatifs soit dans le modèle $1 \mathrm{a}$ soit dans le modèle $1 \mathrm{~b}$, mais pas dans les deux. Dans certaines spécifications, cet effet semble être substitué par la variété intrasectorielle. Cela peut être interprété comme le fait que la variété liée agit également comme une stratégie de portefeuille, mais de manière moins efficace (dans la mesure où les cycles de demande sont davantage corrélés entre les soussecteurs qui se distinguent en-dessous du niveau à deux chiffres). Nous utilisons également le modèle 1 pour ajouter toutes les autres variables une à une. Aucune de ces variables se révèle être significativement liée à la croissance du chômage.

La distinction entre le centre et la périphérie s'avère instructive. Le test de Chow-Wald indique que la distinction entre les régions centrales et périphériques est significative. Ceci résulte principalement des différences d'effet de la densité de population (hautement négative dans les régions du centre et non significative dans les régions périphériques), ce qui suggère que les chômeurs dans la Conurbation profitent de la densité élevée d'un emploi potentiel dans leur région.

\section{Conclusions et implications politiques}

L'objectif de notre étude était d'analyser les effets de la variété sur la croissance économique régionale. Le principal apport de ce travail a consisté à opérer une distinction entre la variété intrasectorielle et la variété intersectorielle. Cette dernière est mesurée au niveau du secteur (classification sectorielle à deux chiffres) alors que la variété intrasectorielle est mesurée au niveau du sous-secteur (au sein des classes à deux chiffres). Nous avons pu constater que les deux variables présentaient des effets très différents sur la productivité, l'emploi et le chômage. Ainsi, les études antérieures ayant mesuré la variété uniquement en termes de ce que nous avons appelé la variété intersectorielle ont ignoré des effets importants de la variété intrasectorielle (Glaeser et al., 1992 ; Acs, 2002). À cet égard, les résultats de ces études devraient être considérés comme moins fiables. 
Plus précisément, nous avons constaté que la variété intrasectorielle favorise la croissance de l'emploi (hypothèse 1) alors que les autres types d'économies d'agglomération ne sont pas significatifs. Sachant que la variété intrasectorielle est principalement présente dans des aires densément peuplées et étant donné que la densité de population n'est pas significativement affectée par la croissance de l'emploi, nous pouvons conclure que les villes ne créent pas automatiquement d'emploi. C'est le caractère diversifié des villes qui est responsable de la création d'emploi. Les résultats montrent aussi que la variété intersectorielle est négativement liée à la croissance du chômage, ce qui signifie que la présence de secteurs non liés dans une région intervient comme un portefeuille contre les chocs de chômage (hypothèse 2), même si dans certaines spécifications, l'effet de la variété liée prend le dessus sur celui de la variété intersectorielle.

À partir de là, et compte tenu des incertitudes statistiques, il en résulte que la politique d'emploi devrait stimuler la variété intrasectorielle quelle qu'elle soit. La variété liée est principalement présente dans les zones urbaines, ce qui fournit une justification pour une politique d'emploi spécifique en milieu urbain. Toutefois, ce serait une erreur de penser que l'agglomération favorise la création d'emploi dans la mesure où la densité de population n'a pas d'effet significatif sur la croissance de l'emploi. La politique devrait plutôt essayer de promouvoir activement le développement des secteurs liés au sein d'une région, par exemple en stimulant le développement de niches haut de gamme dans les secteurs existants et la création de spin-offs d'entreprises installées.

Nos résultats posent aussi la question de la tendance récurrente des décideurs publics de sélectionner a priori des secteurs particuliers et/ou des régions particulières dans leurs objectifs politiques. Les défenseurs d'une telle politique de « choix des gagnants » soutiennent que l'histoire économique met en évidence que la plupart des développements prometteurs dans une économie ont tendance à se regrouper dans un petit nombre de secteurs qui se développent dans des régions spécifiques. Les politiques centrées sur le soutien à la variété intrasectorielle, que nous avons trouvé être la force motrice de la croissance de l'emploi, peuvent être considérées comme une stratégie plus progressive et modeste. D'une part, le risque d'opérer une mauvaise sélection d'activités est réduit parce que l'on considère les compétences régionales existantes comme groupe de construction pour diversifier la base économique de la région. D'autre part, de telles politiques pourraient encore reconnaître le fait que les technologies génériques comme les TIC et les biotechnologies peuvent avoir un impact omniprésent sur le développement économique mais fondamentalement imprévisible au niveau de ses effets spatiaux. 


\section{Références bibliographiques}

ACs Z., 2002 : Innovation and the Growth of Cities. Cheltenham and Northampton MA: Edward Elgar.

ATTARAN M., 1985 : Industrial diversity and economic performance in U.S. areas. The Annals of Regional Science 20, pp. 44-54.

Broersma L. et J. OOSTERHAVEN, 2004 : Regionale Arbeidsproductiviteit: Niveau, groei en verklaring, Report to the Ministry of Economic Affairs of The Netherlands, University of Groningen, Juin 2004.

DISSART J. C., 2003 : Regional economic diversity and regional economic stability: research results and agenda, International Regional Science Review 26, pp. 423-446.

FeldMan M. P. et D. B. AudretsCh, 1999 : Innovation in cities: Sciencebased diversity, specialization and localized competition, European Economic Review, 43(2), Pp. 409-429.

Frenken K., F. G. VAN OORT, T. Verburg et R.A. Boschma, 2004 : Variety and Regional Economic Development in The Netherlands (Den Haag: Ministry of Economic Affairs Research Series), www.minez.nl, 24 decembre.

Glaeser E. L., H. Kallal, J. Scheinkman et A. Shleifer, 1992 : Growth in cities. Journal of Political Economy, 100, pp. 1126-1152.

JACOBS J., 1969 : The Economy of Cities, New York: Vintage.

JACQUEMIN A. P. et C. H. BeRRY, 1979 : Entropy measure of diversification and corporate growth, Journal of Industrial Economics, 27(4), pp. 359-369.

Jaffe A. B., M. Trajtenberg, R. Henderson, 1993 : Geographic localization of knowledge spillovers as evidenced by patent citations. Quarterly Journal of Economics,108(3), pp. 577-598.

KIM S.J., 1997 : Productivity of cities, Aldershot: Ashgate.

KRUGMAN P. R., 1991 : Increasing returns and economic geography, Journal of Political Economy, 99(3), Pp. 483-499.

KRUGMAN P. R., 1993 : Lessons of Massachusetts for EMU, in: F. Torres and F. Giavazzi (eds.), Adjustment and Growth in the European Monetary Union, (Cambridge: Cambridge University Press), pp. 241269.

Los B., 2000 : The empirical performance of a new inter-industry technology spillover measure, pp. 118-151 in: P.P. Saviotti and B. Nooteboom (eds.), Technology and Knowledge, Cheltenham: Edward Elgar. 
I Koen Frenken, Frank G. van Oort et Thijs Verburg

VAN OORT F. G., 2004 : Urban Growth and Innovation. Spatially bounded externalities in the Netherlands, Aldershot: Ashgate.

SAVIOTTI P. P. et A. PYKA, 2004a : Economic development by the creation of new sectors, Journal of Evolutionary Economics, 14(1), pp. 1-35.

SAVIOTTI P. P. et A. PYKA, 2004b : Economic development, variety and employment creation, Revue Economique, 55(6), pp. 1023-1059.

Solow R. M., 1957 : Technical change and the aggregate production function, Review of Economics and Statistics, 39, pp. 312-320.

THEIL H., 1967 : Economics and Information Theory, Amsterdam: NorthHolland. 